04

\title{
Инициация электрического пробоя газа в поле бегущей вдоль металлической поверхности сверхвысокочастотной волны
}

\author{
(С) Л.П. Грачев, И.И. Есаков, А.А. Раваев, Л.Г. Северинов
}

Акционерное общество „Московский радиотехнический институт РАН“, 117519 Москва, Россия

e-mail: grachev@mrtiran.ru

(Поступило в Редакцию 25 декабря 2017 г.)

Описаны результаты экспериментов по электрическому пробою воздуха в линейно-поляризованной бегущей сверхвысокочастотной (СВЧ) волне с глубоко подкритическим уровнем поля. Пробой инициирован линейным электромагнитным вибратором или их системой. Вибраторы расположены над металлической поверхностью, лежащей в плоскости, содержащей волновой вектор СВЧ волны и ее электрическую составляющую. При этом оси вибраторов параллельны этой составляющей поля, и их расстояние до металлической плоскости меньше четверти длины волны поля. В опытах с единичным вибратором это расстояние варьирует. При экспериментах с системой вибраторов они расположены последовательно друг за другом вдоль волнового вектора поля.

DOI: 10.21883/JTF.2018.07.46166.2613

\section{Введение}

Используемый термин сверхвысокочастотная (СВЧ) волна в общем случае подразумевает электромагнитные (ЭМ) волны в диапазоне длин волн $\lambda$ от $10 \mathrm{~m}$ до $0.1 \mathrm{~mm}$, чему соответствуют частоты колебаний $f$ от $30 \mathrm{MHz}$ до $3 \cdot 10^{3} \mathrm{GHz}[1]$. В настоящей работе, являющейся логическим продолжением исследований, представленных в работе [2], имеется в виду преимущественно диапазон дециметровых и сантиметровых $\lambda$. В этом диапазоне в ближней зоне излучающей системы на практике может быть реализован квазиоптический линейно поляризованный СВЧ пучок с ТЕМ структурой поля. Термин квазиоптический подразумевает, что в поперечном волновому вектору $\mathbf{k}$ направлении пучок имеет размеры, не превышающие единиц $\lambda$.

В некоторых практических схемах желательно осуществить электрический разряд в газе, по которому распространяется СВЧ волна. В этом случае амплитуда ее электрической составляющей $E_{0}$ должна быть больше пробойного уровня $E_{\mathrm{br}}$, который в свою очередь, как правило, больше некоего критического уровня $E_{\mathrm{cr}}$. Поле $E_{\mathrm{cr}}$ является минимальным пробойным полем в случае его пространственной однородности и непрерывности. При СВЧ пробое воздуха атмосферного состава и сравнительно высоких газовых давлениях $p$ поле $E_{\text {cr }}$ оценивается по формуле $E_{\mathrm{cr}}=42 p \mathrm{~V} / \mathrm{cm}$, где, как и в последующих аналогичных зависимостях, размерность $p-$ Torr. Эта формула справедлива в рассматриваемом в настоящей статье диапазоне значений $\lambda$ при $p$, больших десятков-сотни Torr. При этих $p$ в образующейся в процессе пробоя воздуха разрядной плазме круговая частота поля $\omega=2 \pi f$ намного меньше частоты столкновений плазменных электронов с молекулами воздуха $v_{c}=4 \cdot 10^{9} \mathrm{p} \mathrm{s}^{-1}$, т. е. нарабатывающаяся плазма является столкновительной [2].
При указанных $\lambda$ и $p$ с помощью доступных СВЧ генераторов практически не удается реализовать в бегущей СВЧ волне условие $E_{0}>E_{\mathrm{br}}$, и электрический разряд в газе необходимо инициировать. В литературе рассмотрены несколько способов инициации [3-5]. Одним из них, например, является вспомогательная реализация в СВЧ пучке импульсного лазерного пробоя газа $[4,5]$.

В работе [6] рассмотрен способ инициации СВЧ пробоя газа путем помещения в ЭМ пучок с глубоко подкритическим уровнем поля $\left(E_{0} \ll E_{\mathrm{cr}}\right)$ параллельно его вектору $\mathbf{E}_{0}$ линейного ЭМ вибратора. Он представляет собой металлический пруток диаметром $2 a \ll(\lambda / 4)$. Длина прутка $2 L$ близка к резонансной длине $2 L_{\text {res }} \approx(\lambda / 2)$, а концы, например, сферически закруглены.

При помещении такого вибратора в пучок бегущей СВЧ волны поле на его полюсах $E_{\text {pol }}$ существенно превышает поле $E_{0}$. В практических схемах при мощности СВЧ пучка $P_{\text {beam }}$ порядка $1 \mathrm{~kW}$ этого может оказаться недостаточно для инициации пробоя газа при высоких давлениях $p$ порядка или больше атмосферного. В этом случае перпендикулярно вектору $\mathbf{k}$ бегущей ЭМ волны может быть помещен плоский металлический лист. При этом амплитуда поля в ближайшей к его поверхности пучности удвоится. Следовательно, при помещении в нее инициирующего вибратора, т.е. на расстоянии от экрана $h=\lambda / 4$, его способность к инициированию пробоя, по крайней мере, удвоится.

В работе [7] приведены экспериментально подтвержденные теоретические соображения по существенному росту отношения $E_{\mathrm{pol}} / E_{0}$ при приближении ЭМ вибратора к экрану, т.е. при $h<\lambda / 4$. В этой работе описаны результаты опытов, в которых это условие выполняется. В них осуществлен пробой воздуха при атмосферном $p$ в ЭМ пучке с $\lambda=12.3 \mathrm{~cm}$ при $P_{\text {beam }} \approx 1 \mathrm{~kW}$ и исходной амплитуде поля $E_{0} \approx 50 \mathrm{~V} / \mathrm{cm}$. В то же время из приве- 
денной выше формулы следует, что при атмосферном $p \approx 760$ Torr поле $E_{\mathrm{cr}} \approx 30 \mathrm{kV} / \mathrm{cm}$. В этой же работе приводятся данные по зажиганию в таком ЭМ пучке при атмосферном $p$ семи глубоко подкритических СВЧ разрядов. Они инициированы линейной системой параллельных $\mathbf{E}_{0}$ ЭМ вибраторов, расположенных над экраном при $h=5 \mathrm{~mm}$.

В настоящей работе описываются эксперименты по инициации пробоя воздуха в бегущей СВЧ волне с глубоко подкритическим уровнем поля с помощью ЭМ вибраторов, помещаемых над металлической плоскостью, не перпендикулярной волновому вектору ЭМ пучка $\mathbf{k}$, а идущей вдоль него и параллельной вектору электрической составляющей поля $\mathbf{E}_{0}$.

\section{Экспериментальные условия}

В экспериментах СВЧ магнетрон мощностью $P_{\text {gen }}=$ $=1 \mathrm{~kW}$ на частоте $f=2.44 \mathrm{GHz}(\lambda=12.3 \mathrm{~cm})$ в одиночных импульсах длительностью $\tau_{\text {pul }}=0.4 \mathrm{~s}$ возбуждает $\mathrm{TE}_{10}$-волну в прямоугольном волноводе сечением $45 \times 90 \mathrm{~mm}$. К выходу волновода герметично пристыкован прямоугольный излучающий рупор с входным сечением $45 \times 90 \mathrm{~mm}$, выходным $-90 \times 90 \mathrm{~mm}$ и длиной $150 \mathrm{~mm}$. СВЧ волна излучается в рабочую камеру. В предположении, что в выходном сечении рупора сохраняется $\mathrm{TE}_{10}$ распределение поля, амплитуда его электрической составляющей в центральной области рупора

$$
E_{0}=(2 / D) \sqrt{P_{\text {gen }} Z_{0}}=136 \mathrm{~V} / \mathrm{cm},
$$

где $D=9 \mathrm{~cm}$ - размер раскрыва рупора и $Z_{0}=$ $=120 \pi \Omega-$ волновое сопротивление вакуума. Давление воздуха $p$ в рабочей камере может устанавливаться в диапазоне от атмосферного значения до $p=10$ Torr и измеряться с точностью \pm 1.5 Torr.

Взаимное расположение помещаемых в излучаемый ЭМ пучок элементов в процессе выполнения экспериментов ясно из приведенной на рис. 1 фотографии. На ней сверху расположен выходной участок рупора. Размер его нижней горизонтальной грани $D=90 \mathrm{~mm}$ может служить масштабом изображений. Волновой вектор излучаемой рупором СВЧ волны $\mathbf{k}$ направлен вертикально вниз. Вектор ее электрической составляющей $\mathbf{E}_{0}$ горизонтален и лежит в плоскости изображения. К дальней на фотографии выходной грани рупора, вдоль которой поле $E_{0}=0$, пристыкован вертикальный металлический лист шириной $120 \mathrm{~mm}$. Снизу этот лист имеет горизонтальную полку, на которую помещен СВЧ поглотитель с конусообразными выступами. Наличие поглотителя обеспечивает режим бегущей волны в излучаемом рупором ЭМ пучке.

На поверхность вертикального листа симметрично относительно оси СВЧ пучка прикреплен пенопластовый радиопрозрачный брусок. Его длина $80 \mathrm{~mm}$, высота над плоскостью листа $32 \mathrm{~mm}$ и ширина $20 \mathrm{~mm}$. В бруске параллельно поверхности листа имеются две системы

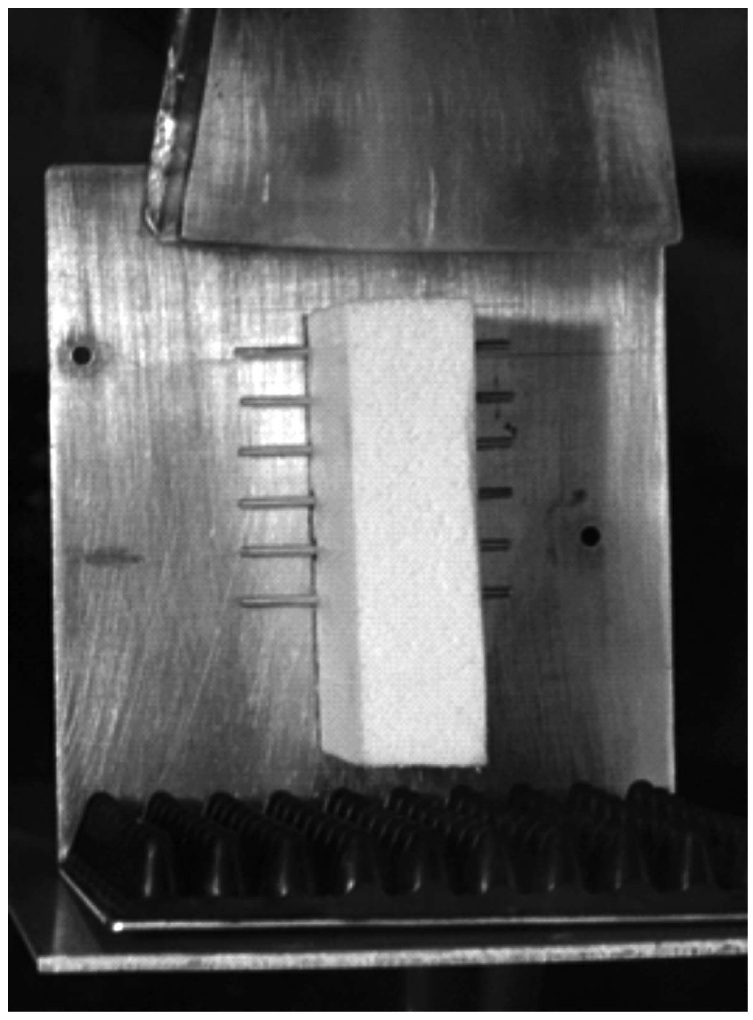

Рис. 1. Взаимное расположение элементов в экспериментальной схеме.

отверстий диаметром $2.1 \mathrm{~mm}$. Первая система, вертикальная относительно плоскости листа, состоит из пяти отверстий, расстояние от которых до выходного сечения рупора равно $30 \mathrm{~mm}$, а расстояния до плоскости листа $h=2.5,8,14.5,20.5$ и $25.5 \mathrm{~mm}$. Вторая система, горизонтальная относительно плоскости листа и вертикальная на фотографии, содержит семь отверстий с одинаковым расстоянием от плоскости листа $h=2.5 \mathrm{~mm}$. Расстояние между ними $\Delta=10 \mathrm{~mm}$. Верхнее отверстие этой системы является нижним для вертикальной системы отверстий.

В эти отверстия могут быть помещены выполненные из алюминиевых прутков диаметром $2 a=2 \mathrm{~mm}$ со сферически закругленными концами ЭМ вибраторы. На приведенной на рис. 1 фотографии это проиллюстрировано вертикальной системой из шести вибраторов.

Для начала развития процесса разряда у концов вибраторов в областях с $E>E_{\mathrm{br}}$ необходимо наличие начальных электронов. Для этого в описываемых экспериментах в качестве вспомогательного источника ультрафиолетового (УФ) излучения использовался синхронизованный с передним фронтом СВЧ импульса высоковольтный разряд длительностью в несколько микросекунд. Этот разряд длиной в несколько миллиметров зажигался между внутренней жилой высоковольтного кабеля и поверхностью листа. Кабель пропускался в видимое на рис. 1 с правой стороны от вибраторов отверстие. 


\section{Результаты экспериментов}

Первоначально эксперименты выполнялись с одним вибратором. В них была определена его длина $2 L$, обеспечивающая инициацию пробоя воздуха при максимальном давлении воздуха $p_{\text {br }}$. Вибратор имел начальную длину $2 L=60 \mathrm{~mm}$. Она лишь на $1.5 \mathrm{~mm}$ меньше размеpa $\lambda / 2$. Этот вибратор был помещен в верхнее отверстие в пенопластовом бруске, соответствующее $h=2.5 \mathrm{~mm}$. В этом случае при постепенной с шагом 15 Torr откачке рабочей камеры вибратор начал инициировать СВЧ пробой воздуха при давлении $p_{\mathrm{br}} \approx 50$ Torr. При этом, как и в последующих опытах, разрядная область реализовывалась только у обращенного к УФ источнику конца вибратора. Затем длина вибратора с шагом, не превышающим $0.5 \mathrm{~mm}$, последовательно уменьшалась. При этом давление $p_{\text {br }}$ увеличивалось, и при $2 L=57 \mathrm{~mm}$ вибратор инициировал СВЧ пробой заполняющего рабочую камеру воздуха уже при атмосферном его давлении. Затем длина вибратора дополнительно уменьшилась на $0.4 \mathrm{~mm}$. Такой вибратор также обеспечивал инициацию пробоя воздуха атмосферного $p$.

В опытах этот же вибратор был помещен и в нижнее отверстие бруска при $h=2.5 \mathrm{~mm}$. При таком расположении в СВЧ пучке он также продолжал инициировать пробой воздуха атмосферного $p$. Во всех последующих экспериментах использовались именно такие инициаторы с $2 L=56.6 \pm 0.1 \mathrm{~mm}$.

В следующей экспериментальной серии вибратор последовательно помещался в вертикальную серию отверстий с различными $h$, и при каждом этом размере определялась величина $p_{\mathrm{br}}$. Полученные экспериментальные результаты приведены на рис. 2.

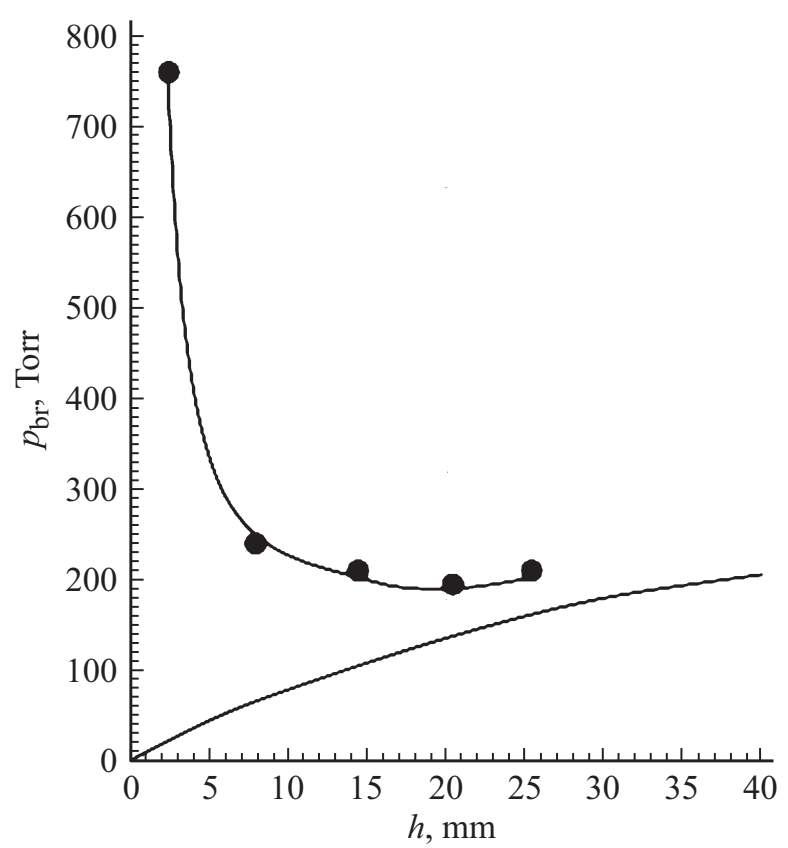

Рис. 2. Результаты экспериментов.

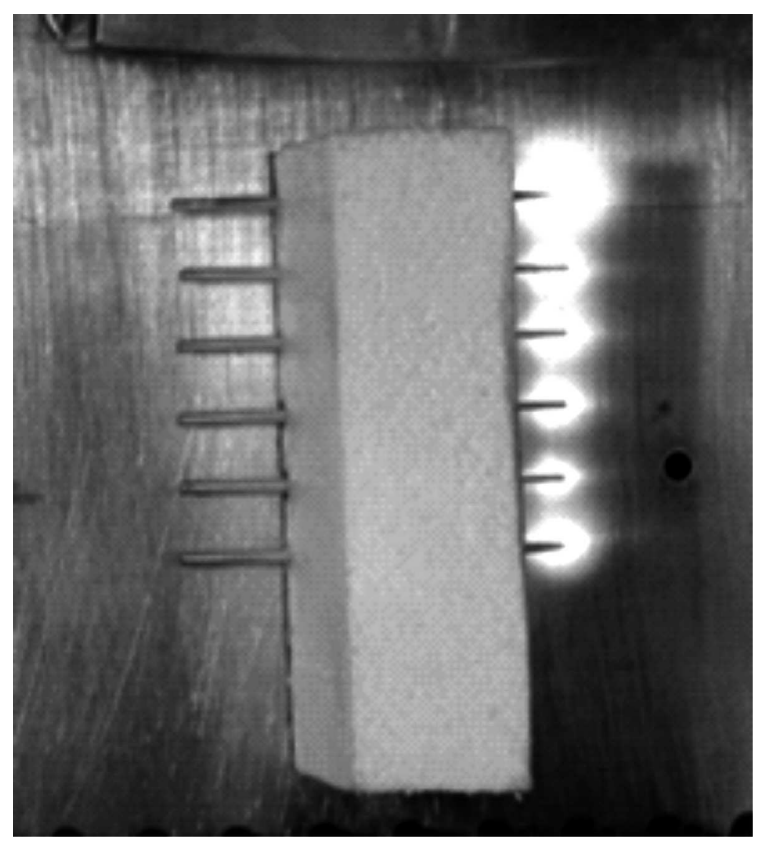

Рис. 3. Внешний вид инициированных ЭМ вибраторами газовых разрядов в СВЧ пучке с глубоко подкритическим уровнем поля.

На рис. 2 экспериментальные значения $p_{\text {br }}$ при различных $h$ соединены аппроксимирующей кривой. На нем дополнительной линией показана условная гармоническая зависимость величины поля СВЧ пучка от расстояния до плоскости листа. Как и следует, возбуждающее вибраторы поле $E_{0}$ равно нулю на проводящей поверхности листа и максимально в центральной осевой области пучка при $h \approx(D / 2)=45 \mathrm{~mm}$. И тем не менее в опытах с уменьшением размера $h$ давление $p_{\text {br }}$ растет. Этот экспериментальный результат практически повторяет результаты опытов при расположении вибратора над проводящей плоскостью, перпендикулярной вектору $\mathbf{k}$ ЭМ пучка [7].

Физическое объяснение этого феномена в обоих случаях одно и то же. Основные активные потери возбуждающей вибратор ЭМ энергии при отсутствии инициированного им СВЧ разряда - это потери на ее переизлучение. Количественно они характеризуются величиной сопротивления излучения $R_{\Sigma}$. При расположении вибратора резонансной длины в поле бегущей СВЧ волны величина $R_{\Sigma}=73 \Omega$ [8]. Она на порядки превышает активное сопротивление материала вибратора $R_{\sigma}$ и задает сравнительно малую величину его электрической добротности $Q$. Последняя и определяет величину отношения $E_{\mathrm{pol}} / E_{0}$. Из развитой в работе [7] теории следует, что при уменьшении размера $h$ величина $R_{\Sigma}$ уменьшается, и добротность $Q$ эквивалентной электрической схемы вибратора как колебательного контура лавинообразно растет. В результате это и компенсирует падение величины поля $E_{0}$. 
В заключительной экспериментальной серии исследовалась возможность реализации системы СВЧ разрядов в поле бегущей ЭМ волны. Из предварительных соображений ясно, что взаимное электродинамическое влияние вибраторов будет минимальным при их близком расположении к проводящей плоскости, т.е. малом $h$. Именно из этих соображений вертикальная система отверстий в диэлектрическом бруске и была выполнена при $h=2.5 \mathrm{~mm}$. В опытах последовательно увеличивалось количество вибраторов, начиная с помещения первого вибратора в ближайшее к излучающему рупору отверстие. Затем количество вибраторов $N$ последовательно увеличивалось, и определялась возможность одновременной инициации ими СВЧ пробоя воздуха при атмосферном $p$. В опытах в каждом СВЧ импульсе разряд зажигался только до $N=6$. При наличии седьмого вибратора все верхние вибраторы инициировали пробой воздуха, а нижний - нет. На рис. 3 приведена фотография горящей линейки из шести разрядов.

В данных опытах, как и во всех предыдущих, первоначально использовалась вспомогательная УФ подсветка вибраторов. Контрольные эксперименты показали, что при наличии системы вибраторов, начиная с $N=5$, УФ подсветка уже не требовалась. Рис. 3 и соответствует горению разрядов без предварительной УФ подсветки.

Следующая из фотографии различная интенсивность горения разрядов связана, в первую очередь, с их расстоянием до излучающего раскрыва рупора. На ее величину может сказываться и продольная неоднородность величины поля вдоль СВЧ пучка. Она может быть как начальной, так и обусловленной факторами, связанными со взаимным электродинамическим влиянием вибраторов.

\section{Заключение}

Таким образом, эксперименты показали, что инициированный линейным ЭМ вибратором глубоко подкритический СВЧ разряд может быть зажжен в квазиоптическом СВЧ пучке при помещении вибратора над проводящей плоскостью, параллельной волновому вектору ЭМ пучка. При этом, как и при помещении вибратора над плоскостью, перпендикулярной волновому вектору, расстояние его до проводящей плоскости должно быть существенно меньше четверти длины волны СВЧ поля. В обеих этих схемах в СВЧ пучке возможна реализация системы из нескольких разрядов.

Полученные результаты открывают возможности для применения такого вида СВЧ разряда в практических схемах при использовании широко распространенных СВЧ генераторов с малым уровнем выходной мощности масштаба сотен W. При этом встанет требующий предварительной проработки вопрос крепления вибраторов к поверхности экрана. Почти очевидно, что при увеличении расстояния между вибраторами в их линейной системе может возрасти и количество одновременно зажигаемых разрядов. С точки зрения применения такого вида разряда, например в работе [9], описаны результаты экспериментов, демонстрирующие принципиальную возможность поджига им пропан-воздушной горючей смеси в ее высокоскоростном потоке. Система разрядов в поле бегущей СВЧ волны может осуществить практически мгновенный мультиочаговый поджиг такой смеси, например, в трубчатой камере сгорания.

\section{Список литературы}

[1] Лебедев И.В. Техника и приборы СВЧ. М.: Высшая школа, $1970.439 \mathrm{c}$.

[2] Грачев Л.П., Есаков И.И., Александров К.В., Раваев А.А., Северинов Л.Г., Ходатаев К.В. Газовый электрический разряд в квазиоптическом СВЧ пучке. М.: АО МРТИ РАН, 2015. $187 \mathrm{c}$.

[3] Богатов Н.А., Быков Ю.В., Венедиктов Н.П. и др. // Физика плазмы. 1986. Т. 12. № 6. С. 725.

[4] Батанов Г.М., Грищинин С.И., Коссый И.А. и др. // Труды ФИАН. 1985. Т. 160. С. 174.

[5] Зарин А.С., Кузовников А.А., Шибков В.М. Свободно локализованный СВЧ-разряд в воздухе. М.: Нефть и газ, 1996. $204 \mathrm{c}$

[6] Грачев Л.П., Есаков И.И., Мишин Г.И., Ходатаев К.В. // ЖТФ. 1995. Т. 65. Вып. 7. С. 60-67.

[7] Грачев Л.П., Есаков И.И., Лавров П.Б., Раваев А.А. // ЖТФ. 2012. Т. 82. Вып. 2. С.73-78.

[8] Драбкин А.Л., Зузенко В.Л., Кислов А.Г. Антенно-фидерные устройства. М.: Советское радио, 1974. 536 с.

[9] Александров К.В., Грачев Л.П., Есаков И.И., Раваев А.А., Северинов Л.Г., Яковлев А.Ю. // ЖТФ. 2016. Т. 86. Вып. 4. C. 28-33. 